\title{
Motor and cognitive function in Lewy body dementia: comparison with Alzheimer's and Parkinson's diseases
}

\author{
Kanna K Gnanalingham, E Jane Byrne, Andrew Thornton, Mike A Sambrook, \\ Paul Bannister
}

\begin{abstract}
Objective-Motor and cognitive function were compared in patients with Lewy body dementia, Parkinson's disease, or Alzheimer's disease, to identify features that may be clinically useful in differentiating Lewy body dementia from Alzheimer's disease and Parkinson's disease.
\end{abstract}

Methods-A range of neuropsychological function and extrapyrimidal signs (EPS) was assessed in 16 patients with Lewy body dementia, 15 with Parkinson's disease, 25 with Alzheimer's disease, and 22 control subjects.

Results-The severity of total motor disability scores increased in the following order: controls $\approx$ Alzheimer's disease $<$ Parkinson's disease < Lewy body dementia. Compared with patients with Parkinson's disease, patients with Lewy body dementia had greater scores for rigidity and deficits in the finger tapping test, but rest tremor and left/right asymmetry in EPS were more evident in Parkinson's disease. Patients with Lewy body dementia were also less likely to present with left/right asymmetry in EPS at the onset of their parkinsonism. "Sensitivity" to neuroleptic drugs was noted in $33 \%$ of patients with Lewy body dementia. Alzheimer's disease and Lewy body dementia groups had greater severity of dementia compared with the Parkinson's disease group and controls. Neuropsychological evaluation disclosed severe but similar degrees of impaired performances in tests of attention (digit span), frontal lobe function (verbal fluency, category, and Nelson card sort test) and motor sequencing in both Lewy body dementia and Alzheimer's disease groups, than Parkinson's disease and controls. In the clock face test, improved performance was noted in the "copy" compared to "draw" part of the test in controls, patients with Alzheimer's disease, and those with Parkinson's disease, but not in the patients with Lewy body dementia, who achieved equally poor scores in both parts of the test.

Conclusions-EPS in Lewy body dementia resemble those seen in idiopathic Parkinson's disease, although less rest tremor and left/right asymmetry but more severe rigidity favours a diagnosis of Lewy body dementia. The unique profile of patients with Lewy body dementia seen in the clock face test suggests that this simple and easy to adminster test may be useful in the clinical setting to differentiate Lewy body dementia and Alzheimer's disease.

$(\mathcal{F}$ Neurol Neurosurg Psychiatry 1997;62:243-252)

Keywords: Lewy body dementia; Alzheimer's disease; extrapyramidalism; neuropsychology

Lewy body dementia is increasingly being recognised as a common cause of dementia in elderly people, probably the second most common type after Alzheimer's disease, in postmortem studies. ${ }^{12}$ Histologically, numerous Lewy bodies in the brain stem (substantia nigra and locus coeruleus), subcortical (nucleus basalis of Meynert), and cortical regions have been noted in Lewy body dementia. ${ }^{3-5}$ Correspondingly, there are neuronal losses and gliosis in the substantia nigra, locus coeruleus, and nucleus basalis of Meynert, with associated loss of dopamine in the basal ganglia and choline acetyl transferase in the cerebral cortex..$^{3-10}$ In Lewy body dementia, increased numbers of cortical senile plaques have been described, but the density of neurofibrillary tangles is insufficient to meet the diagnostic criteria for Alzheimer's disease.

Clinically, Lewy body dementia is characterised by progressive dementia with often a fluctuating course, extrapyramidal signs (EPS), hallucinations, and increased sensitivity to neuroleptic drugs. ${ }^{1451112}$ Patients with Lewy body dementia may initially present with cognitive impairment or EPS. Recently, operational criteria have been proposed for the clinical diagnosis of Lewy body dementia. ${ }^{13} 14$ The diagnostic criteria were validated against pathologically confirmed cases of Alzheimer's disease, multi-infarct dementia, and Lewy body dementia and were reported to have high diagnostic specificity $(90 \%-97 \%)$, sensitivity $(80 \%-85 \%)$, and interrater reliability (agreement in $78 \%-94 \%$ of cases). ${ }^{15} 16$

Although EPS is one of the features recognised in the diagnostic criteria for Lewy body dementia, there is discrepancy over its prevalence. Some authors have reported EPS in almost all cases of Lewy body dementia, with over $70 \%$ qualifying for a second diagnosis of Parkinson's disease at some stage of the illness. ${ }^{51117}$ By contrast, others have noted only mild EPS in Lewy body dementia.469 Furthermore, most of the motor features of Parkinson's disease have been described in 
Lewy body dementia, although again there is considerable variation between studies. ${ }^{1}$ The reasons for this are not clear. It may in part reflect sampling bias, with some studies including only patients with a clinical diagnosis of Alzheimer's disease, ${ }^{69}$ whereas others have recruited patients from several specialties. ${ }^{11}$

Comparison of the EPS of idiopathic Parkinson's disease and Lewy body dementia may identify features that would be helpful in the antemortem diagnosis of Lewy body dementia. The only study so far to examine this issue reported that bradykinesia was slightly more common in Lewy body dementia $(86 \%$ of cases) than Parkinson's disease $(56 \%),{ }^{18}$ tremor and rigidity being equally likely in both diseases. Asymmetry in these motor signs was not assessed. Moreover, studies to date have assessed EPS in Lewy body dementia by retrospective review of case notes, which vary considerably in the clinical detail provided. Also the assessment was carried out by clinicians who were not blind to the diagnosis of the patients. Thus the findings of these studies are open to observer bias. Quantitative methods to assess EPS (for example, measurement of speed of walking), which would also overcome observer bias, were not carried out.

Furthermore, when the initial presentation of Lewy body dementia is one of impaired cognition, it can mimic Alzheimer's disease and be difficult to differentiate at the onset of the illness. ${ }^{69}$ Neuropsychological tests may prove a useful clinical aid in such cases. But the neuropsychological profile of Lewy body dementia is far from clear and to date only a few studies have investigated this issue. Hansen $e t a l^{9}$ compared the neuropsychological performance of a group of patients with Lewy body dementia and patients with Alzheimer's disease, matched for age and overall severity of dementia. The Lewy body dementia group performed less well than the patients with Alzheimer's disease in tests of attention (digit span), visuospatial processing (block design and copy a cross tests, which are thought to reflect in part parietal lobe function), and semantic memory (verbal fluency and category tests, which are thought to reflect frontal lobe function). Both groups did equally well in tests of memory (visual and auditory). However, the patients with Lewy body dementia assessed in this study were from a pool of patients clinically diagnosed to have Alzheimer's disease and therefore may not be representative of Lewy body dementia in general. ${ }^{9}$

Others have employed computer controlled neuropsychological testing batteries to show greater impairment in components of visual memory and attentional ability in clinically diagnosed cases of Lewy body dementia compared with patients with Alzheimer's disease. ${ }^{19-21}$ Interestingly, in one of the tests for attention (visual search matching to sample), impairment was seen only in Lewy body dementia and not Alzheimer's disease. ${ }^{19}$ This suggests that there may be qualitative as well as quantitative differences in the neuropsycho- logical profiles of patients with Lewy body dementia and patients with Alzheimer's disease. This is an important finding, but its usefulness in the day to day clinical setting is likely to be limited by the complex nature of this test.

Therefore, in the present study we proposed to (a) extensively characterise EPS in Lewy body dementia, Parkinson's disease, and Alzheimer's disease, by observer rating EPS and quantitatively assessing walking and speed of finger movement; $(b)$ compare the performance of these groups on a range of neuropsychological tasks, to identify qualitative as well as quantitative differences in the cognitive impairment of Alzheimer's disease and Lewy body dementia. The neuropsychological tests were chosen on the basis that they were sensitive to frontal and temporoparietal lobe dysfunction (as is thought to occur in Lewy body dementia) and because these tests are simple and can be made use of in the clinical setting.

\section{Methods}

\section{PATIENTS}

A consecutive series of patients attending the Departments of Old Age Psychiatry, Geriatrics, and Neurology were recruited for this study. The inclusion criteria were $(a)$ between 60 to 90 years of age; (b) a clinical diagnosis of idiopathic Parkinson's disease based on the Parkinson's disease United Kingdom Parkinson's disease Society Brain Bank (SBB) criteria ${ }^{22}$; (c) a clinical diagnosis of probable or possible Alzheimer's disease according to the National Institute of Neurological and Communicative Disorders and Stroke and the Alzheimer's disease and Related Disorders Association (NINCDSADRDA) criteria ${ }^{23}$; (d) a clinical diagnosis of Lewy body dementia according to both the Newcastle $^{14}$ and Nottingham criteria ${ }^{13} ;(e)$ a Hachinski ${ }^{24}$ ischaemic score $\leqslant 5$. A group of healthy subjects between the ages of 60 to 90 and with no history of psychiatric or neurological disorders was also recruited from the carers of the study population.

Informed consent was obtained from the patients or their carers. Both the patients and controls were assessed during an interview lasting up to 90 minutes. None of the patients were tested during a period of episodic confusion. Patients on antiparkinsonian treatments were assessed before taking their morning medications, so that their motor disability could be determined after an overnight drug free period. Hospital and general practitioner's case notes were later reviewed to obtain further information on past medical and surgical history, a record of medications taken, results of investigations, and the mode of presentation of current illness.

\section{NEUROLOGICAL ASSESSMENT}

The EPS were assessed with the unified Parkinson's disease rating scale (UPDRS) ${ }^{25}$ This scale has several sections, comprising (a) mentation, behaviour, and mood (items 1-4; total score $=16$ ); $(b)$ activities of daily living 
(items 5-17; total score $=52$ ); (c) motor examination (items 18-31; total score $=56$ ); (d) complications of therapy (items 32-42; total score $=23$ ). For each section, a number of items were scored. Individual item scores ranged from 0 to 4 and higher scores represented greater impairment. Both the frequency and severity of EPS were noted.

Bradykinesia was also quantitatively assessed by counting the number of times the patient could alternatively tap two keys situated $20 \mathrm{~cm}$ apart in 30 seconds. ${ }^{26}$ Walking was quantitatively assessed by asking the patient to rise from an armless chair, walk $6 \mathrm{~m}$, return, and sit down. The time taken to accomplish this task and the number of footsteps taken were noted and these two values were multiplied to give an index of walking ability in step-seconds. ${ }^{27}$

PSYCHIATRIC ASSESSMENT

Mini mental state examination (MMSE)

This is an 11 item examination, proved to be a quick and reliable test of cognitive function. ${ }^{28}$ Of a possible total score of 30 , a score $\leqslant 23$ was considered to indicate cognitive impairment in an elderly population.

\section{Clinical dementia rating (CDR)}

The CDR scale is a global rating scale for dementia. ${ }^{29}$ The final score is heavily influenced by the score for the memory category. In this scale dementia is graded as 0 none, 0.5 questionable, 1 mild, 2 moderate, and 3 severe dementia.

Cornell scale for depression in dementia (CSDD) This is a 19 item interviewer administered scale that uses information from interviews with both the patient and their carers. ${ }^{30}$ The CSDD scale is especially suited for assessing depression in patients with dementia (maximum score 38 ).

\section{NEUROPSYCHOLOGICAL ASSESSMENT Digit span}

This test assesses auditory attention and short term memory. ${ }^{31}$ For assessment of the digit span forward, the patient was asked to repeat a string of random numbers in the exact order given. For assessment of digit span reverse, the patient was asked to repeat a string of random numbers in reverse order. The string of numbers was read out by the examiner at a rate of $1 / \mathrm{s}$.

\section{Verbal and category fluency}

These tests assess access to semantic information under time constraint and are sensitive to frontal lobe damage. ${ }^{32}{ }^{33}$ In the verbal fluency task, the patients were requested to generate as many words as possible that began with the letters $\mathrm{S}, \mathrm{A}$, and $\mathrm{F}$, excluding names, places, and different forms of the same word. For each letter, one minute was given to generate the words and the total number of words generated with all three letters was noted. In the category fluency task, the patients were asked to generate as many animals as possible in one minute.
Clock face test

This test examines executive and visuospatial functioning and includes two parts. ${ }^{34}$ First, in the "draw" part the patient was instructed to "draw the face of a clock, showing the numbers and the hands set to 10 after 11 ". Then, in the "copy" part, the patient was instructed to copy the correctly drawn clock face. No time limit was placed on this task. Performance on both "draw" and "copy" parts of this test were rated on a 1 to 10 scale by an examiner blind to the clinical diagnosis of the patient. Preliminary results of this test have been reported elsewhere. ${ }^{35}$

\section{Motor sequencing tasks}

The "fist-edge-palm" and the Ozeretski tests for the presence of motor dyspraxia were used, as described previously. ${ }^{36}$ In both tests, the patient's performance was scored as $0=$ normal response; 1 = patient was able to learn and repeat the test sequence at least once but not six times consecutively; $2=$ patient unable to satisfactorily carry out the sequence even once. The cumulative score (0-4) was used as an index of performance in the motor sequencing tasks.

\section{Nelson card sort test}

This test assesses the patient's ability to develop new concepts and shift sets and it has been shown to be sensitive to frontal lobe damage. ${ }^{37}$ The patient's performance on this test was assessed in terms of (a) number of categories achieved; $(b)$ total errors; $(c)$ perseverative errors; $(d)$ percentage of perseverative errors (perseverative errors as a \% of total errors).

\section{STATISTICAL ANALYSIS}

In all tests, the null hypothesis was rejected at a significance level of $\mathbf{P}<0.05$. Parametric data were analysed, with or without transformation (logarithms), by one way or repeated measures analysis of variance (ANOVA) and post hoc Duncan's multiple range test. Scores on the CSDD and UPDRS motor section were entered as covariates. Non-parametric data were analysed by Kruskal Wallis and post hoc Mann-Whitney $U$ tests. Nominal data (for example, sex) were analysed by $\chi^{2}$ test with Yates' correction.

\section{Results}

DEMOGRAPHIC FINDINGS (TABLE 1)

In total 56 patients and 22 controls were recruited for the study. Of the 25 patients with Alzheimer's disease, there were 22 probable and three possible cases according to the NINCDS-ADRDA criteria. ${ }^{23}$ All 16 patients with Lewy body dementia conformed to the Newcastle criteria and Nottingham criteria for probable disease. ${ }^{1314}$ Nine of the patients with Lewy body dementia (56\%) initially presented with EPS, five $(31 \%)$ presented with concurrent EPS and cognitive changes, and two (13\%) presented first with cognitive impairment. There were no differences in age, sex, number of years of education, and social class 
Table 1 Demographic and psychiatric findings

\begin{tabular}{|c|c|c|c|c|c|}
\hline & Controls & $A D$ & $P D$ & $L B D$ & $F$ or $H$ ratios \\
\hline No of patients & 22 & 25 & 15 & 16 & \\
\hline $\begin{array}{l}\text { Age (y) } \\
\text { (range) }\end{array}$ & $\begin{array}{l}73 \cdot 3(1 \cdot 3) \\
(62-86)\end{array}$ & $\begin{array}{l}75 \cdot 7(1 \cdot 4) \\
(63-85)\end{array}$ & $\begin{array}{l}72 \cdot 6(2 \cdot 1) \\
(60-85)\end{array}$ & $\begin{array}{l}76 \cdot 4(1 \cdot 8) \\
(61-88)\end{array}$ & $F=1.3 ; P=0.3$ \\
\hline Duration of illness $(y)^{a}$ & & $3 \cdot 7(0 \cdot 4)$ & $9 \cdot 2(2 \cdot 0) t$ & $6 \cdot 3(1 \cdot 1)$ & $F=6.3 ; P<0.01$ \\
\hline Sex $(\% \text { male })^{b}$ & 60 & 47 & 67 & 50 & \\
\hline Education $(y)^{\mathrm{a}}$ & $10 \cdot 1(0.5)$ & $9.9(0.4)$ & $10.3(0.9)$ & $11 \cdot 0(1 \cdot 1)$ & $F=0.5 ; P=0.7$ \\
\hline Social class ( $\%$ class III) & & 44 & 40 & & $\mathrm{H}=1.4 ; \mathrm{P}=0.7$ \\
\hline$\underset{\text { (range) }}{\text { MMSE (total score) }}$ & $\begin{array}{l}29 \cdot 1(0 \cdot 3) \\
(24-30)\end{array}$ & $\begin{array}{l}13 \cdot 4(1 \cdot 6)^{\star \star} \\
(1-28)\end{array}$ & $\begin{array}{l}24 \cdot 1(1 \cdot 3)^{\star}+t \\
(16-30)\end{array}$ & $\begin{array}{l}12 \cdot 5(1 \cdot 8)^{\star \star} \neq \ddagger \\
(0 \cdot 26)\end{array}$ & $F=37 ; P<0.0001$ \\
\hline $\mathrm{CDR}^{\mathrm{b}}$ & $0(0-0)$ & $1(0 \cdot 5-3)^{\star \star}$ & $0(0-1)^{\star}+\dagger$ & $1.5(0.5-3)^{\star \star} \neq \ddagger$ & $\mathrm{H}=60 ; \mathrm{P}<0.0001$ \\
\hline $\mathrm{CSDD}^{\mathrm{b}}$ & $1.0(0-10)$ & $2 \cdot 0(0-17)^{\star}$ & $3 \cdot 5(0-12)^{\star \star}$ & $6 \cdot 0(2-20)^{\star \star}+\neq \ddagger$ & $H=18 ; P<0.001$ \\
\hline $\begin{array}{l}\text { Thought disorder } \\
\text { (UPDRS item 2) }\end{array}$ & $0(0-0)$ & $0(0-3)^{\star}$ & $0(0-2)$ & $3(0-4)^{\star \star}+十 \neq \ddagger$ & $\mathrm{H}=43 ; \mathrm{P}<0.0001$ \\
\hline
\end{tabular}

"Values are means (SEM). One way ANOVA and post hoc Duncan's multiple range test.

"Values are medians (range). Kruskal Wallis and post hoc Mann-Whitney $U$ tests.

Post hoc comparisons: ${ }^{\star} \mathrm{P}<0.05 ; \star \star \mathrm{P}<0.01 v$ controls. $+\mathrm{P}<0.05 ;++\mathrm{P}<0.01 v \mathrm{AD}$. $\neq \ddagger \mathrm{P}<0.01 v \mathrm{PD}$.

$\mathrm{AD}=$ Alzheimer's disease; PD = Parkinson's disease; LBD = Lewy body dementia; MMSE = mini mental state examination; CDR = clinical dementia rating; $\mathrm{CSDD}=$ Cornell scale for depression in dementia.

between groups (table 1). The Parkinson's disease group had a longer duration of illness than the group with Alzheimer's disease $(\mathrm{P}<0 \cdot 05)$.

\section{PSYCHIATRIC FINDINGS (TABLE 1)}

There were corresponding changes in MMSE and CDR scores between the groups. Scores for MMSE decreased in the following order: controls > Parkinson's disease $>>$ Alzheimer's disease $\approx$ Lewy body dementia. Scores for CDR increased in the following order: controls < Parkinson's disease $<$ Alzheimer's disease $\approx$ Lewy body dementia. There were no differences in the MMSE $(P=0.7)$ and CDR scores $(P=0.6)$ between the Alzheimer's disease and Lewy body dementia groups. Further analysis of the 11 individual items that make up the MMSE disclosed that in general, controls and patients with Parkinson's disease performed better than those with Alzheimer's disease or Lewy body dementia in all of the tasks (data not shown). There were no significant differences between the performances of Alzheimer's disease and Lewy body dementia groups in any of the items that make up the MMSE. The CSDD scores increased in the following order: controls < Alzheimer's disease $\approx$ Parkinson's disease < Lewy body dementia. Scores for thought disorder (item 2 of UPDRS) were higher for the group with Lewy body dementia than the other groups $(P<0.01)$.

NEUROLOGICAL FINDINGS

EPS noted at the time of interview (table 2)

Four of the controls (18\%), 18 patients with Alzheimer's disease (72\%), 16 with Lewy body dementia (100\%), and 15 with Parkinson's disease $(100 \%)$ had isolated EPS (defined as score $>1$ on any of the items on the motor section of the UPDRS). Three patients with Alzheimer's disease (12\%), 15 with Lewy body dementia (94\%), and 15 with Parkinson's disease (100\%) had parkinsonism according to the SBB criteria. ${ }^{22}$ None of the controls had parkinsonism.

The total UPDRS motor scores increased in the following order: controls $\approx$ Alzheimer's disease $<<$ Parkinson's disease < Lewy body dementia (table 2). Further analysis of the items making up the motor section of UPDRS showed that scores for tremor at rest, action tremor, rigidity, bradykinesia, postural stability, and falls were greater for the Parkinson's disease and Lewy body dementia groups compared with controls and patients with Alzheimer's disease $(P<0.05$; table 2$)$. Scores for these motor signs were also greater for patients with Alzheimer's disease than controls

Table 2 EPS noted at the time of interview

\begin{tabular}{|c|c|c|c|c|c|}
\hline & $\begin{array}{l}\text { Controls } \\
(n=20)\end{array}$ & $\begin{array}{l}A D \\
(n=22-25)\end{array}$ & $\begin{array}{l}P D \\
(n=15)\end{array}$ & $\begin{array}{l}L B D \\
(n=12-16)\end{array}$ & $F, H$, or $\chi^{2}$ ratios \\
\hline $\begin{array}{l}\text { UPDRS: }{ }^{\text {Urange) }}\end{array}$ & $\begin{array}{l}1 \cdot 0(0 \cdot 2) \\
(0-3)\end{array}$ & $\begin{array}{l}5 \cdot 9(1) \\
(1-20)\end{array}$ & $\begin{array}{l}29 \cdot 5(4)+t^{\star \star} \\
(5-49)\end{array}$ & $\begin{array}{l}38 \cdot 3 \text { (3) } \ddagger \ddagger \dagger+\star \star \\
(12-50)\end{array}$ & $F=68 ; P<0.0001$ \\
\hline Tremor at rest (item 20) & $0(0-0)$ & $0(0-0 \cdot 5)$ & $2(0.5-4)+t^{\star \star}$ & $1.25(0-4) t^{\star \star}$ & $\mathrm{H}=53 ; \mathrm{P}<0.0001$ \\
\hline Action tremor (item 21) ${ }^{\mathrm{b}}$ & $0(0-0)$ & $0(0-2)^{\star}$ & $1(0-3)+t^{\star \star}$ & $1(0-4)+t^{\star \star \star}$ & $\mathrm{H}=27 ; \mathrm{P}<0.0001$ \\
\hline Rigidity (item 22) ${ }^{\mathrm{b}}$ & $0(0-1)$ & $1(0-2 \cdot 5)^{\star \star}$ & $3(1-4)+\dagger^{\star \star}$ & $4(1 \cdot 5-4) \ddagger \dagger \dagger^{\star \star}$ & $\mathrm{H}=57 ; \mathrm{P}<0.0001$ \\
\hline \multicolumn{6}{|l|}{ Hand and feet bradykinesia } \\
\hline (items 23-26) $^{\mathrm{b}}$ & $0(0-3)$ & $1(0-4 \cdot 5)^{\star \star}$ & $7(1-16)+t^{\star \star}$ & $12(2-16)+t^{\star \star}$ & $\mathrm{H}=55 ; \mathrm{P}<0.0001$ \\
\hline Postural stability (item 30) ${ }^{\mathrm{b}}$ & $0(0-2)$ & $1(0-3)^{\star \star}$ & $2(0-4) \dagger^{\star \star}$ & $3(1-4)+t^{\star \star}$ & $\mathrm{H}=39 ; \mathrm{P}<0.0001$ \\
\hline Body bradykinesia (item 31$)^{\mathrm{b}}$ & $0(0-1)$ & $0(0-2)^{\star \star}$ & $2(0 \cdot 5-4)+t^{\star \star}$ & $3 \cdot 25(0 \cdot 5-4) t^{\star \star}$ & $\mathrm{H}=57 ; \mathrm{P}<0.0001$ \\
\hline Falls (item 13) & $0(0-0 \cdot 5)$ & $0(0-1)^{\star \star}$ & $1(0-2 \cdot 5)+f^{\star \star}$ & $1.75(0-4) f t^{\star \star}$ & $\mathrm{H}=47 ; \mathrm{P}<0.0001$ \\
\hline \multicolumn{6}{|l|}{ Asymmetry of motor signs: } \\
\hline Left/right asymmetry & $1(4 \cdot 5)$ & $1(4)$ & $12(80)+t^{\star \star}$ & $4(25) \ddagger \dagger$ & $\chi^{2}=37 ; \mathrm{P}<0.0001$ \\
\hline Upper/lower limb asymmetry & $2(9)$ & $10(40)^{\star}$ & $11(73) \dagger^{\star \star}$ & $9(56)^{\star \star}$ & $\chi^{2}=17 ; P<0.001$ \\
\hline \multicolumn{6}{|l|}{ Tapping test $($ taps $/ 30 \mathrm{~s}):^{\mathrm{a}}$} \\
\hline Dominant hand & $84 \cdot 5(3)$ & $56 \cdot 0(5)^{\star \star}$ & $47 \cdot 7(7)^{\star \star}$ & $19 \cdot 5(5) \neq \neq+\dagger^{\star \star}$ & $\mathrm{F}=20 ; \mathrm{P}<0.0001$ \\
\hline Non-dominant hand & $82 \cdot 2(3)$ & $51 \cdot 9(5)^{\star \star}$ & $44 \cdot 9(7)^{\star \star}$ & $16 \cdot 6(5) \neq \neq \dagger \dagger \star \star \star$ & $F=24 ; P<0.0001$ \\
\hline \multicolumn{6}{|l|}{ Walking test: ${ }^{\mathrm{d}}$} \\
\hline Time taken (s) & $11 \cdot 9(11-13)$ & $18 \cdot 7(15-24)$ & $41 \cdot 3(23-75)+t^{\star \star}$ & $59 \cdot 2(38-93) t^{\star \star}$ & $\mathrm{F}=15 ; \mathrm{P}<0.0001$ \\
\hline Number of steps & $17 \cdot 3(15-20)$ & $22 \cdot 4(19-26)$ & $41 \cdot 3(26-65) \dagger \dagger^{\star \star}$ & $50.9(39-66) f f^{\star \star}$ & $F=14 ; P<0.0001$ \\
\hline Step-seconds & $217(192-246)$ & $408(283-586)$ & $1686(596-4802)+f^{\star \star}$ & $3103(1544-6195)+t^{\star \star}$ & $F=16 ; P<0.0001$ \\
\hline
\end{tabular}

"Values are means (SEM). One way ANCOVA, with the CSDD scores as covariates and post hoc Duncan's multiple range test.

"Values are medians (range). Kruskal Wallis and post hoc Mann-whitney $U$ tests.

Values are number of patients $(\%)$ with asymmetric motor symptoms. $\chi^{2}$ test.

dValues are means ( $95 \%$ confidence interval). One way ANCOVA, with the CSDD scores as covariates and post hoc Duncan's multiple range test. The data were analysed after logarithmic transformation and consequently the $95 \%$ confidence intervals are given rather than the SEM.

Post hoc comparisons: ${ }^{*} \mathrm{P}<0.05 ;{ }^{\star} \mathrm{P}<0.01 v$ controls; $+\mathrm{P}<0.05 ;+\uparrow \mathrm{P}<0.01 v \mathrm{AD} ; \ddagger \mathrm{P}<0.05 ; \neq \neq \mathrm{P}<0.01 v \mathrm{PD}$. 
Table 3 EPS noted at the onset of parkinsonism in Parkinson's disease (PD) and Lewy body dementia (LBD)

\begin{tabular}{lcll}
\hline & $\begin{array}{l}P D(n=11) \\
n(\%)\end{array}$ & $\begin{array}{l}L B D(n=12) \\
n(\%)\end{array}$ & $\chi^{2}$ test \\
\hline Tremor at rest & $9(82 \%)$ & $8(67 \%)$ & $\chi^{2}=0 \cdot 9 ; \mathrm{P}=0 \cdot 4$ \\
Rigidity & $9(82 \%)$ & $9(75 \%)$ & $\chi^{2}=0 \cdot 1 ; \mathrm{P}=0 \cdot 8$ \\
Bradykinesia & $7(64 \%)$ & $7(58 \%)$ & $\chi^{2}=0 \cdot 1 ; \mathrm{P}=0 \cdot 8$ \\
Asymmetry of motor symptoms: & $11(100 \%)$ & $5(42 \%)^{\star \star}$ & $\chi^{2}=9 \cdot 2 ; \mathrm{P}<0 \cdot 01$ \\
$\quad$ Left/right asymmetry & $8(73 \%)$ & $6(50 \%)$ & $\chi^{2}=0 \cdot 8 ; \mathrm{P}=0 \cdot 4$ \\
\hline Upper/lower limb asymmetry & & &
\end{tabular}

$\star \star \mathrm{P}<0.01 v \mathrm{PD} ; \chi^{2}$ test.
Parkinson's disease performed less well in the walking test than the control or Alzheimer's disease groups, as shown by the greater time taken, number of steps, and step-seconds $(\mathrm{P}<0.01 ;$ table 2$)$.

EPS noted at the onset of parkinsonism in Parkinson's disease and Lewy body dementia (table 3)

Retrospective review of case notes showed adequate clinical details on the nature of EPS at the onset of parkinsonism in 11 patients with Parkinson's disease and 12 patients with Lewy body dementia. There were no differences in the proportion of patients with Parkinson's disease or with Lewy body dementia who presented with tremor, rigidity, or bradykinesia. Left/right asymmetry in motor signs was noted in a greater proportion of patients with Parkinson's disease $(100 \%)$ than Lewy body dementia $(42 \% ; P<0.01)$. There were no differences in the proportion of patients with Parkinson's disease or Lewy body dementia presenting with asymmetry in EPS between the upper and lower limbs. dist were assessed in the motor section of the UPDRS (data there were no significant differences between the Parkinson's disease and Lewy body dementia groups, except for tremor at rest, which was noted in a greater proportion of patients with Parkinson's disease (93\%) than with Lewy body dementia ( $50 \% ; P<0.01)$.

A greater proportion of patients with Parkinson's disease $(80 \%)$ compared with other groups had left/right asymmetry in motor signs $(P<0.05$; table 2$)$. Asymmetry of motor signs between upper and lower limbs was noted in a greater proportion of patients with Alzheimer's disease (40\%), Lewy body dementia (56\%), or Parkinson's disease (73\%) than controls $(9 \%)(P<0.05)$.

Patients in the Alzheimer's disease, Parkinson's disease, and Lewy body dementia groups performed less well in the tapping test (taps/30 s) than the controls, with both the dominant and non-dominant hands $(P<0.01$; table 2). The Lewy body dementia group performed less well than patients with Alzheimer's disease and patients with Parkinson's disease in this respect $(P<0.01)$. Patients with Lewy body dementia or

\section{NEUROPSYCHOLOGICAL FINDINGS (TABLE 4; FIGURE)}

Digit span forward and reverse of patients with Alzheimer's disease and patients with Lewy body dementia was less than that for controls and Parkinson's disease groups $(P<0.01$; table 4). The total number of words generated in the verbal fluency and category tests by the Lewy body dementia groups was less than the controls $(P<0.05$; table 4$)$. In this respect, the Alzheimer's disease and Lewy body dementia groups performed less well than the Parkinson's disease group $(P<0.01)$. The total error scores in the motor sequencing tasks of the three disease groups were greater than controls $(P<0.05$; table 4$)$. The total error scores for the Alzheimer's disease and Lewy body dementia groups were greater than the Parkinson's disease group $(P<0.01)$. The Lewy body dementia and more especially the Alzheimer's disease groups performed less well in the Nelson card sort test than the control and Parkinson's disease groups, as shown by Alzheimer's disease, Parkinson's disease, and

Table 4 Neuropsychological findings

\begin{tabular}{|c|c|c|c|c|c|}
\hline & $\begin{array}{l}\text { Controls } \\
(n=22)\end{array}$ & $\begin{array}{l}A D \\
(n=25)\end{array}$ & $\begin{array}{l}P D \\
(n=13)\end{array}$ & $\begin{array}{l}L B D \\
(n=16)\end{array}$ & $F$ or $H$ ratios \\
\hline \multicolumn{6}{|l|}{ Digit span:a } \\
\hline Forward & $6 \cdot 2(0 \cdot 3)$ & $4 \cdot 2(0 \cdot 4)^{\star \star}$ & $6 \cdot 1(0 \cdot 4) \mathrm{tt}$ & $3.7(0.5) \neq \ddagger^{\star \star}$ & $F=11 ; P<0.0001$ \\
\hline Reverse & $4.0(0.3)$ & $2 \cdot 2(0 \cdot 4)^{\star \star}$ & $4.2(0.5) \mathrm{tt}$ & $2 \cdot 1(0 \cdot 4) \neq f^{\star \star}$ & $F=8 ; P<0.0001$ \\
\hline Verbal fluency (total words: S, A, F) & $32 \cdot 1(3)$ & $9 \cdot 2(2)^{\star \star}$ & $21 \cdot 0(5) \mathrm{tt}^{\star}$ & $8 \cdot 3(2)+f^{\star \star}$ & $F=14 ; P<0.0001$ \\
\hline Category fluency (animals in $1 \mathrm{~min}$ ) & $14 \cdot 1(1)$ & $5 \cdot 1(1)^{\star \star}$ & $10.9(2)+t^{\star}$ & $4 \cdot 9(1)=f^{\star \star}$ & $F=18 ; P<0.0001$ \\
\hline Motor sequencing tasks ${ }^{\mathrm{b}}$ & $0(0-1)$ & $2.5(0-4)^{\star \star}$ & $0(0-3 \cdot 5) t^{\star}$ & $4(0-4) \neq \ddagger^{\star \star}$ & $\mathrm{H}=40 ; \mathrm{P}<0.0001$ \\
\hline Nelson card sort: ${ }^{2}$ & $(n=21)$ & $(n=15)$ & $(n=12)$ & $(n=5)$ & \\
\hline Categories & $5 \cdot 0(0.5)$ & $1.9(0.4)^{\star \star}$ & $4 \cdot 1(0 \cdot 8) \dagger$ & $2 \cdot 7(1 \cdot 1)^{\star}$ & $F=6.8 ; P<0.001$ \\
\hline Total error & $12 \cdot 2(2)$ & $25 \cdot 2(3)^{\star}$ & $17 \cdot 7(5)$ & $22 \cdot 0(5)^{\star \star}$ & $F=3.6 ; P<0.05$ \\
\hline Perseverative errors & $5 \cdot 2(1)$ & $17 \cdot 7(3)^{\star \star}$ & $12.9(4)$ & $11 \cdot 2(6)$ & $F=4.7 ; P<0.01$ \\
\hline$\%$ Perseverative errors & $29 \cdot 4(4)$ & $58.9(6)^{\star}$ & $36 \cdot 0(11)$ & $46 \cdot 4(15)$ & $F=3.9 ; P<0.05$ \\
\hline Clock face test ${ }^{c}$ & $(n=20)$ & $(n=24)$ & $(n=11)$ & $(n=16)$ & \\
\hline Draw part & $8 \cdot 2(0 \cdot 2)$ & $3.7(0.5)^{\star \star}$ & $6 \cdot 2(0 \cdot 7) t^{\star}$ & $2 \cdot 4(0 \cdot 4) \neq \ddagger^{\star \star}$ & \\
\hline Copy part & $9 \cdot 6(0 \cdot 1)$ & $5.5(0.7) \iint^{\star \star}$ & $7 \cdot 1(0.8) \oint t^{\star}$ & $2 \cdot 4(0 \cdot 6) \dagger f \neq 7^{\star \star}$ & \\
\hline
\end{tabular}

Values are means (SEM). One way ANCOVA, with the CSDD scores as covariates and post hoc Duncan's multiple range test.

bValues are medians (range). Kruskal Wallis and post hoc Mann-whitney $U$ tests.

Values are means (SEM). Repeated measures ANCOVA, with the CSDD $(t=-1 \cdot 8 ; \mathrm{P}=0 \cdot 1)$ and UPDRS total motor scores $(t=-2 \cdot 2 ; \mathrm{P}<0 \cdot 05)$ as covariates and post hoc Duncan's multiple range test. There were significant effects for group $(\mathrm{F}=17 ; \mathrm{P}<0.0001)$, “draw"/“copy" (F = $31 ; \mathrm{P}<0.0001)$ and interaction terms and post hoc Duncan's

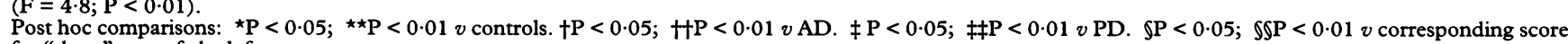
for "draw" part of clock face test. 
Typical drawings produced on the clock face test by (A) controls, (B) patients with Alzheimer's disease, (C) patients with Parkinson's disease, and (d1 and d2) patients with Lewy body dementia. These consisted of "draw" and "copy" parts. ${ }^{34}$ Patient performance was rated on a 1 to 10 scale and the scores are given next to each drawing. For the controls, patients with Alzheimer's disease, and patients with Parkinson's disease, the scores were in general greater in the "copy" than the "draw" parts of the test $(A-C)$. For the Lewy body dementia group, the scores for the "copy" part of the test were in general the same (as shown in d1) or less (d2) than that for the "draw" part.

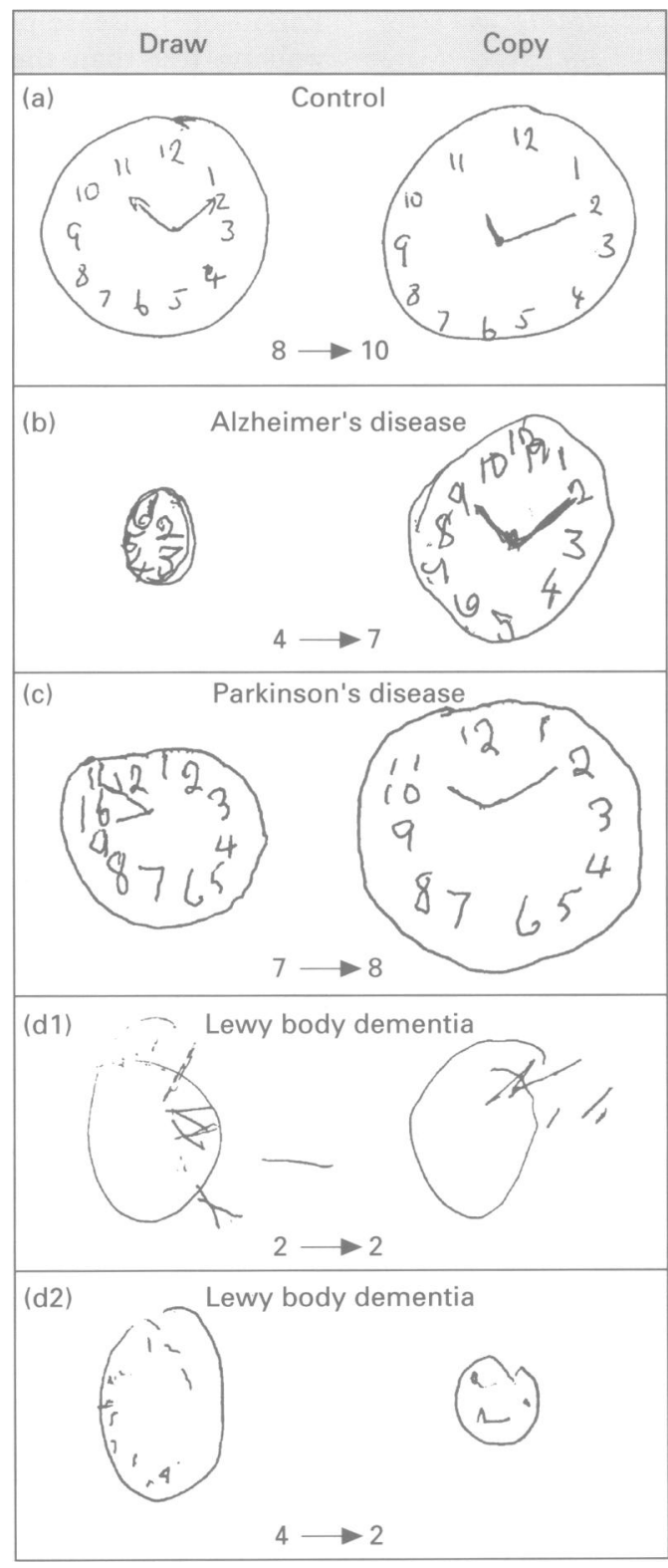

fewer categories achieved, greater total errors and perseverative errors, and $\%$ perseverative errors (table 4).

For analysis of the clock face test, the UPDRS motor scores were entered as covariates to compensate for poor performance resulting from motor disability. The repeated measures ANCOVA showed that in the "draw" part of this test the scores of the groups decreased in the following order: control $>$ Parkinson's disease $>$ Alzheimer's disease $\approx$ Lewy body dementia (table 4). In the "copy" part of the test, the scores decreased in the following order: control > Parkinson's disease $\approx$ Alzheimer's disease > Lewy body dementia. For the controls, patients with Alzheimer's disease, and patients with Parkinson's disease, the scores were greater in the "copy" than "draw" parts of the test $(P<0.05$; table 4 and figure). The scores for the "copy" and "draw" parts of the test were the same for the Lewy body dementia group. Using the criteria that a diagnosis of Lewy body dementia equates to "draw" scores being greater than "copy" scores, the clock face test was noted to have high specificity (98\%), positive predictive value $(80 \%)$ and negative predictive value $(82 \%)$, but low sensitivity $(25 \%)$ and misclassification rates $(18 \%)$. Using the criteria of "draw" scores being less than or equal to "copy" scores improved the sensitivity (88\%), but at the expense of lower specificity $(67 \%)$.

\section{MEDICATIONS TAKEN BY SUBJECTS}

At the time of examination, one patient with Alzheimer's disease, two with Parkinson's disease, and one with Lewy body dementia were taking antidepressant drugs. Six patients with Alzheimer's disease were also taking neuroleptic drugs. There were no significant differences in the UPDRS motor scores between the patients with Alzheimer's disease taking neuroleptic drugs (mean $(S E M)=7 \cdot 1(1)$ ) and those not taking them (5.6 (1); $P=0 \cdot 5)$. Two patients with Lewy body dementia occasionally required neuroleptic drugs for sedation at night. Nine patients with Alzheimer's disease and nine with Lewy body dementia received neuroleptic drugs at some point during their illness. Of these, severe adverse reactions to neuroleptic drugs (marked EPS) were noted in three $(33 \%)$ patients with Lewy body dementia.

At the time of examination, 14 patients with Parkinson's disease and 10 patients with Lewy body dementia were on antiparkinsonian medication, comprising levodopa $(n=24)$, selegiline $(n=12)$, apomorphine infusions ( $n=$ $2)$, and pergolide $(n=1)$. One of the patients with Parkinson's disease had a history of poor compliance with levodopa. All of the patients with Parkinson's disease $(n=15)$ and the 12 patients with Lewy body dementia on whom levodopa had been tried at some point during their illness were found to be responsive to levodopa (reduced EPS on levodopa). There were no differences in the drug free interval for antiparkinsonian medications between the Parkinson's disease mean (SEM) $12 \cdot 8$ (2) hours) and Lewy body dementia groups (12.6 (1) hours; $P=0.9$ ). There were no differences between the Parkinson's disease and Lewy body dementia groups in the UPDRS scores for complications arising from antiparkinsonian therapy $(8.3$ (2) and $7 \cdot 6$ (1) respectively; $P=0.7$ ) and activities of daily living (24.8 (3) and 29.3 (2) respectively; $P=$ $0 \cdot 2)$.

\section{Discussion}

From the outset, it is important to note that cases of Lewy body dementia, Alzheimer's disease, and Parkinson's disease were clinically diagnosed in this study. Although pathologically confirmed cases would be ideal, the clinical criteria employed have been validated against pathologically confirmed cases, and are reported to have high sensitivity, specificity, and, importantly, positive predictive value (the probability of a clinically diagnosed patient having pathological confirmation of disease is $82 \%$ for Parkinson's disease, $88 \%$ for Alzheimer's disease, and $95 \%$ for Lewy body dementia). ${ }^{162238}$ 
EPS AND NEUROPATHOLOGICAL CORRELATES EPS were common in Lewy body dementia. They were noted in all cases examined in this study and $94 \%$ of patients with Lewy body dementia qualified for a second diagnosis of Parkinson's disease. Moreover, patients in the Lewy body dementia group had greater UPDRS total motor scores, more severe rigidity, and poorer performance in the tapping test, than the Parkinson's disease group. Classic resting tremor and left/right asymmetry in EPS were evident in a smaller proportion of patients with Lewy body dementia than patients with Parkinson's disease. Thus about six years after the onset of their illness, the patients with Lewy body dementia showed, in effect, late stages of parkinsonism-an asymmetric, akinetic rigid syndrome. Subtle differences in EPS such as less motor asymmetry, resting tremor, and speed of finger movement, but more severe rigidity may be of limited help in differentiating established cases of Lewy body dementia from Parkinson's disease.

In the present study, $93 \%$ of patients with Parkinson's disease and $63 \%$ of patients with Lewy body dementia were taking antiparkinsonian medications at the time of interview. It can therefore be argued that the greater UPDRS motor score of the Lewy body dementia group was due to a smaller proportion of patients with Lewy body dementia receiving levodopa. We attempted to minimise the effects of antiparkinsonian therapy by interviewing such patients after an overnight drug free period. A longer drug free interval was deemed ethically inappropriate. Moreover, closer inspection of the UPDRS total motor scores disclosed that on average, patients with Lewy body dementia on antiparkinsonian treatment had higher motor scores $(42 \cdot 9)$ than those not receiving any $(30.3)$. A similar pattern was also evident in the Parkinson's disease group, in which one patient failed to take any medication due to poor compliance. This suggests that after an overnight drug free state, antiparkinsonian medications have little if any effect on motor disability in this group of patients and therefore the differences in the UPDRS motor scores of Lewy body dementia and Parkinson's disease groups were genuine.

A review of the published literature disclosed that whereas EPS is a recognised feature of Lewy body dementia, there is controversy over its prevalence. Although some authors have reported that EPS is common and can be severe in Lewy body dementia, ${ }^{51117}$ others have noticed only mild EPS. ${ }^{49}$ Likewise there is considerable variation in the reported prevalence of different types of EPS. Nevertheless, in agreement with the present findings, a recent review of 75 published case reports of Lewy body dementia noted that rigidity was more often reported than other EPS such as tremor. ${ }^{1}$ The only study to date to attempt a direct comparison of EPS in Lewy body dementia and idiopathic Parkinson's disease found greater bradykinesia and a tendency for less resting tremor in Lewy body dementia. ${ }^{18}$ Like previous studies, asymmetry in EPS was not reported. Whereas we agree with the finding on resting tremor, a non-significant trend towards greater bradykinesia in Lewy body dementia was found in the present study. However, the two studies are not directly comparable, as Louis et $a l^{18}$ only included patients with Lewy body dementia presenting at the onset of their illness with parkinsonism. This is likely to introduce sampling bias.

Indeed, one of the reasons for the discrepancy over EPS in Lewy body dementia in the published literature may be sampling bias. In the studies by Hansen et $a l^{9}$ and Forstl et $a l^{6}$ only patients with a clinical diagnosis of Alzheimer's disease were included, which may underlie the low prevalence of EPS noted in these studies. On the other hand, studies such as ours, in which patients were recruited from neurology, geriatrics, and psychiatry included a greater number of patients with Lewy body dementia presenting with parkinsonism. ${ }^{511}$ Furthermore, unlike the present study, in previous studies EPS was assessed by retrospective review of case notes, which are of varying quality and quantitative measurements of the various types of EPS that would be less open to observer bias were not carried out. These factors may also contribute to the inconsistent findings made on EPS to date.

To further characterise the nature of EPS in Lewy body dementia, the type of EPS noted at the onset of parkinsonism in patients with Parkinson's disease and patients with Lewy body dementia was assessed by retrospective review of case notes. This showed that patients with Lewy body dementia were less likely to present with left/right asymmetry in EPS than patients with Parkinson's disease, although resting tremor, bradykinesia, and rigidity were equally likely in both groups. Evidently, parkinsonism at the onset of Lewy body dementia may resemble that seen at the onset of idiopathic Parkinson's disease, as has been noted previously. ${ }^{11}$ Nevertheless, as with established cases of Lewy body dementia, a lack of motor asymmetry at the onset of parkinsonism seems to favour a diagnosis of Lewy body dementia. But the quantitative nature of this finding limits its clinical use.

In the present study, all patients with Lewy body dementia and Parkinson's disease showed some degree of response to levodopa (when tried) and had similar scores for complications arising from antiparkinsonian treatments. Response to levodopa has been noted in a majority of patients with Lewy body dementia taking it. ${ }^{111839}$ Some studies have reported a lack of levodopa response, although this may also reflect failure to try a sufficiently high dose of levodopa in Lewy body dementia. ${ }^{4041}$ Indeed, in Lewy body dementia relatively high doses of levodopa may not be tolerated as this may worsen confusion and psychosis.

The frequent appearance of EPS and its responsiveness to levodopa are important features of Lewy body dementia that are concordant with the Parkinson's disease-like neuropathological changes reported in this 
disorder. Thus in Lewy body dementia there are Lewy bodies, neuronal losses, and gliosis in the substantia nigra, the pathological hallmark of Parkinson's disease. ${ }^{34}$ This is associated with greatly reduced concentrations of dopamine in the basal ganglia of patients with Lewy body dementia, ${ }^{10}$ which suggest that as with Parkinson's disease, dopaminergic insufficiency is likely to underlie the onset of motor disability in Lewy body dementia. The pronounced sensitivity to neuroleptic drugs noted in some patients with Lewy body dementia, both in the present study and by others, may also reflect this state of dopamine deficiency and therefore increased susceptibility to dopamine receptor blockade. ${ }^{12}$

\section{NEUROPSYCHOLOGY AND}

NEUROPATHOLOGICAL CORRELATES

Several neuropsychological tests were carried out to establish the nature of cognitive impairment in Lewy body dementia and to identify specific tasks that differentiate it from Alzheimer's disease. It was evident that components of the widely used MMSE do not distinguish between Alzheimer's disease and Lewy body dementia. Similarly, both groups of patients, irrespective of the severity of dementia, were equally impaired in tests of digit span, verbal fluency, category, motor sequencing, and Nelson card sort test.

By contrast, patients with Lewy body dementia showed a different profile of performance on the clock face test to the other groups. Not only did the Lewy body dementia group score poorly in the "draw" part, but they also failed to exhibit the improvement in scores on the "copy" paradigm which was found with Alzheimer's disease, Parkinson's disease, and control groups. The qualitative nature of this finding may be of use clinically as a screening instrument. Indeed, the clock face test used as a diagnostic test for Lewy body dementia was noted to have high specificity $(98 \%)$, positive likelihood ratio $(12 \cdot 5)$, and positive predictive value $(80 \%)$, but low sensitivity (25\%) (Lewy body dementia was diagnosed in cases with greater "draw" than "copy" scores). Nevertheless, despite its low sensitivity, those cases detected by this test are very likely to have Lewy body dementia and only one patient with Parkinson's disease erroneously tested positive in the clock face test. Thus the clock face test may be a useful clinical aid as a quick and simple method to differentiate Alzheimer's disease and Lewy body dementia in the clinical setting.

It is important to note that the differential performance of patients with Lewy body dementia in the clock face test is unlikely to be attributable to the intake of levodopa, greater scores for motor disability, or depression, factors that can affect neuropsychological function. ${ }^{42}$ To minimise such confounding influences, the neuropsychological tests were carried out after an overnight drug free period and both UPDRS motor scores and CSDD ratings were entered as covariates in the statistical analysis. Moreover, patients with Parkinson's disease, despite exhibiting severe
EPS and also receiving levodopa, had a different profile of performance on the clock face test compared with the Lewy body dementia group. This suggests that the differences in the neuropsychological tasks reflect differential impairments in cognitive function between groups.

The clock face test has been used previously in patients with Lewy body dementia as part of a pilot study investigating the effect of levodopa on cognitive function. ${ }^{39}$ But, only the "draw" paradigm was tested in this study and no firm conclusions were reached due to the small sample size $(n=5)$ and the considerable variability in cognitive function in Lewy body dementia. ${ }^{39}$ The clock face test assesses executive and visuospatial functioning, which are therefore greatly impaired in Lewy body dementia. ${ }^{34} \mathrm{~A}$ severe visuospatial dysfunction in Lewy body dementia has been noted previously, as assessed by the block design and copy a cross tests. ${ }^{9}$ These authors also noted greater deficiencies in digit span and verbal and category fluency in patients with Lewy body dementia than in patients with Alzheimer's disease. This is not consistent with the findings of the present study and the reasons for this discrepancy are uncertain. Some caution needs to be exercised when comparing the results of the two studies. The cases of Lewy body dementia assessed in the study by Hansen et $a l^{9}$ were from a group of patients who were clinically diagnosed as having Alzheimer's disease and at postmortem fulfilled the pathological criteria for both Alzheimer's disease and Lewy body dementia. Such patients may therefore represent a mixed Alzheimer's disease/Lewy body dementia group and may not be characteristic of Lewy body dementia in general, in which Alzheimer's disease type neuropathology is minimal. Nevertheless, subtle differences in the impairment of visual memory and attention have been described between patients with Lewy body dementia and those with Alzheimer's disease in studies utilising computer controlled neuropsychology test batteries. ${ }^{19-21}$ In one of the tests for attentional ability, patients with Lewy body dementia but not patients with Alzheimer's disease performed worse than controls. ${ }^{19}$ This further confirms the presence of qualitative differences in the cognitive impairment of patients with Alzheimer's disease and patients with Lewy body dementia.

The neuropathological correlates for cognitive impairment seen in Lewy body dementia is not clear. The severity of dementia in Lewy body dementia was noted to correlate with cortical Lewy body densities in one study ${ }^{3}$ but not in another. ${ }^{4}$ Impaired visuospatial function can be seen in dementia syndromes of cortical and subcortical types. ${ }^{43}$ In Lewy body dementia there is dysfunction of the subcortical dopaminergic system affecting the brainstem and basal ganglia and this may underlie the poor visuospatial ability of these patients, as apparent in the clock face test. However, the unusual severity of this deficit may reflect additional cortical dysfunction in this disorder. 
In Lewy body dementia there is greater loss of cholinergic neurons in the nucleus basalis of Meynert and choline acetyltransferase in the cerebral cortex than in Alzheimer's disease. ${ }^{91044}$ Thus the cognitive impairment in Lewy body dementia may be a combination of cortical and subcortical types.

Only mild cognitive deficits were apparent in the Parkinson's disease group as evident by the marginally lower MMSE and verbal fluency scores than in the control group. Impaired verbal fluency is characteristic of subcortical dementia and has been noted in Parkinson's disease. ${ }^{42}$ Although motor deficits are the predominant feature in Parkinson's disease, about $20 \%$ of patients with Parkinson's disease may eventually become demented, which is twofold to threefold greater than in the rest of the population. ${ }^{45}$ Interestingly, bilateral EPS in Parkinson's disease is associated with greater cognitive decline some years later. ${ }^{46}$ This parallels the current findings on Lewy body dementia, as a dementia syndrome with relatively symmetric EPS, both at the onset and later in the course of the illness. Whether patients with Parkinson's disease with symmetric motor signs represent early cases of Lewy body dementia is not clear. Nevertheless, symmetry in motor disability seems to be associated with cognitive decline in both disorders.

\section{Conclusions}

It is evident from this study that there are similarities as well as differences in the motor and cognitive function of Lewy body dementia in comparison with Alzheimer's disease and Parkinson's disease. The subtle differences in the nature of EPS between Lewy body dementia and Parkinson's disease may be of limited help in clinically differentiating the two disorders. Cognitive impairment in Lewy body dementia was pronounced and mimicked Alzheimer's disease, but there were qualitative differences in the performances of these groups on the clock face test. This simple, quick, and easy to administer test may prove useful in the clinical setting to distinguish Lewy body dementia and Alzheimer's disease. Correct antemortem diagnosis of Lewy body dementia is important in its effective management, as these patients may be sensitive to neuroleptic drugs, which carry considerable morbidity and mortality in this disorder. ${ }^{12}$

The motor disability of Lewy body dementia is consistent with the dopaminergic dysfunction in the brain stem. The cognitive impairment in the disease is probably attributable to both the subcortical dopaminergic and cortical cholinergic dysfunction. Differences in the severity of cortical (producing cognitive impairment) and brain stem pathology (producing mainly EPS), may be responsible for the heterogeneity in the clinical presentation of Lewy body dementia.

We gratefully acknowledge the helpful suggestions and assistance of Dr S Simpson, Ms C Donaldson, Ms J Winters, Dr P Gibson, and Professor A Burns. Statistical advice was provided by $\mathrm{Mr} \mathrm{B}$ Faragher.
1 Lennox G. Lewy body dementia. Bailliere's Clin Neurol 1992;1:653-76.

2 McKeith IG, Galasko D, Wilcock GK, Byrne JE. Lewy body dementia-diagnosis and treatment. Br f Psychiatry 1995;167:708-17.

3 Lennox G, Lowe J, Landon M, Byrne EJ, Mayer RJ, Godwin-Austen RB. Diffuse Lewy body disease: correlative neuropathology using anti-ubiquitin immunocytochemistry. I Neurol Neurosurg Psychiatry 1989;52: $1236-47$.

4 Perry RH, Irvin D, Blessed G, Fairbairn A, Perry EK. Senile dementia of the Lewy body type: a clinically and neuropathologically distinct form of Lewy body dementia in the elderly. F Neurol Sci 1990;95:119-39.

5 Gibb WRG, Luthert PJ, Janota I, Lantos PL. Cortical Lewy body dementia: clinical features and classification. f Neurol Neurosurg Psychiatry 1989;52:185-92.

6 Forstl H, Burns A, Luthert P, Cairns N, Levy R. The Lewy body variant of Alzheimer's disease: clinical and pathological findings. Br F Psychiatry 1993;162:385-92.

7 Lippa CF, Smith TW, Swearer JM. Alzheimer's disease and Lewy body disease: a comparative clinicopathological study. Ann Neurol 1994;35:81-8.

8 Dickson DW, Davies P, Mayeux R, Crystal H, Horoupian DS, Thompson A, Goldman JE. Diffuse Lewy body disease: neuropathological and biochemical studies in 6 patients. Acta Neuropathologica 1987;75:8-15.

9 Hansen L, Salmon D, Galasko D, Masliah E, Katzman R, DeTeresa $R$, et al. The Lewy body variant of Alzheimer's disease: a clinical and pathologic entity. Neurology 1990; disease: $1-8$.

10 Langlais PJ, Thal L, Hansen L, Galasko D, Alford M, Masliah E. Neurotransmitters in basal ganglia and cerebral cortex of Alzheimer's disease, with and without Lewy bodies. Neurology 1993;43:1927-934.

11 Byrne EJ, Lennox G, Lowe J, Godwin-Austen RB Diffuse Lewy body disease: clinical features in 15 cases. $f$ Neurol Neurosurg Psychiatry 1989;52:709-17.

12 McKeith IG, Fairbairn A, Perry R, Thompson P, Perry E. Neuroleptic sensitivity in patients with senile dementia of Lewy body type. $B M \mathcal{F}$ 1992;305:673-8.

13 Byrne EJ, Lennox GG, Godwin-Austen RB, Jefferson D, Lowe J, Mayer RJ, et al. Dementia associated with cortical Lewy bodies: proposed clinical diagnostic criteria. Lewy bodies: proposed

14 McKeith IG, Perry RH, Fairbairn AF, Jabeen S, Perry EK. Operational criteria for senile dementia of Lewy body type (SDLT). Psychol Med 1992;22:911-22.

15 McKeith IG, Fairbairn AF, Bothwell RA, Moore PB, Ferrier IN, Thompson P, Perry RH. An evaluation of the predictive validity and inter-rater reliability of clinical diagnostic criteria for senile dementia of Lewy body type. Neurology 1994;44:872-7.

16 McKeith IG, Fairbairn AF, Perry RH, Thompson P. The clinical diagnosis and misdiagnosis of senile dementia of Lewy body type (SDLT). Br $\mathcal{F}$ Psychiatry 1994;165: 324-32.

17 Kosaka K. Diffuse Lewy body disease in Japan. $\mathcal{F}$ Neurol 1990;237:197-204.

18 Louis ED, Goldman JE, Powers JM, Fahn S. Parkinsonian features of eight pathologically diagnosed cases of diffuse Lewy body disease. Mov Disord 1995;10:188-94.

19 Sahgal A, Galloway PH, McKeith IG, Edwardson JA, Lloyd S. A comparative study of attentional deficits in senile dementias of Alzheimer and Lewy body types. Dementia 1992;3:350-4.

20 Sahgal A, Galloway PH, McKeith IG, Lloyd S, Cook JH, Ferrier NI, Edwardson JA. Matching-to-sample deficits in patients with senile dementias of Alzheimer and Lewy body types. Arch Neurol 1992;49:1043-6.

21 Galloway P, Sahgal A, McKeith IG, Lloyd S, Cook JH, Ferrier NI, Edwardson JA. Visual pattern recognition memory and learning deficits in senile dementias of Alzheimer and Lewy body types. Dementia 1992;3:101-7.

22 Hughes AJ, Daniel SE, Kilford L, Lees AJ. Accuracy of clinical diagnosis of idiopathic Parkinson's disease:a clinico-pathological study of 100 cases. $\mathcal{F}$ Neurol Neurosurg ico-pathological study of

23 McKhann G, Drachman D, Folstein M, Katzman R, Price D, Stadlan EM. Clinical diagnosis of Alzheimer's disease: Report of the NINCDS-ADRDA work group under ease: Report of the NINCDS-ADRDA work group under
the auspices of Department of Health and Human the auspices of Department of Health and Human Services Task Forc

24 Hachinski VC, Iliff LD, Zilhka E, Du Boulay GH, McAllister VL, Marshall J, et al. Cerebral blood flow in dementia. Arch Neurol 1975;32:632-37.

25 Lang AE, Fahn S. Assessment of Parkinson's disease. In: Munstat TL, ed. Quantification of neurologic deficit. London: Butterworths: 1989:285-309.

26 Merello M, Sabe L, Teson A, Migliorelli R, Petracchi M, Leiguarda $R$, Starkstein $S$. Extrapyramidalism in Alzheimer's disease: prevalence, psychiatric and neuropsychological correlates. 7 Neurol Neurosurg Psychiatry 1994;57:1503-9.

27 Webster DD. Critical analysis of the disability in Parkinson's disease. Modern Treatment 1968;5:257-82.

28 Folstein MF, Folstein SE, McHugh PR. Mini-mental state: a practical method for grading the cognitive state of
patients for the clinician. $尹$ Psychiatr Res $1975 ; 12: 189-98$. 29 Morris JC. The Clinical Dementia Rating (CDR): current version and scoring rules. Neurology 1993;43:2413-4.

30 Alexopoulos GS, Abrams RC, Young RC, Shamoian CA. Cornell scale for depression in dementia. Biol Psychiatry 1988;23:271-84. 
31 Weschler D. Wechsler adult intelligence scale manual. New York: Psychological Corporation, 1955.

32 Borkowski JG, Benton AL, Spreen O. Word fluency and brain damage. Neuropsychology 1967;5:135-40.

33 Benton AL. Differential behavioural effects in frontal lobe disease. Neuropsychology 1968;6:53-60

34 Libon DJ, Swenson RA, Barnoski EJ, Sands LP. Clock drawing as an assessment tool for dementia. Arc Clin Neuropsychol 1993;8:405-15.

35 Gnanalingham KK, Byrne EJ, Thornton A. Clock-face drawing to differentiate Lewy body and Alzheimer type dementia syndromes. Lancet 1996;347:696-7.

36 Buchanan RW, Heinrichs DW. The neurological evaluation scale (NES): a structured instrument for the assessment of neurological signs in schizophrenia. Psychiatry ment of neurological

37 Nelson HE. Modified card sorting test sensitive to frontal lobe defects. Cortex 1976;12:313-24.

38 Burns A, Luthert P, Levy R, Jacoby R, Lantos P. Accuracy of clinical diagnosis of Alzheimer's disease. BMF 1990; 301:1026.

39 Williams SW, Byrne EJ, Stokes P. The treatment of diffuse Lewy body disease: a pilot study. International fournal of Geriatric Psychiatry 1993;8:731-9.
40 Mark MH, Sage JI, Dickson DW, Schwarz KO, Duvoisin RC. Levodopa-non responsive Lewy body parkinsonism: clinicopathologic study of two cases. Neurology 1992;42: 1323-7.

41 Hughes AJ, Daniel SE, Blankson S, Lees AJ. A clinicopathological study of 100 cases of Parkinson's disease. Arch Neurol 1993;50:140-8.

42 Gotham AM, Brown RG, Marsden CD. Frontal cognitive function in patients with Parkinson's disease "on" and "off" levodopa. Brain 1988;111:299-321.

43 Cummings JL. Subcortical dementia: neuropsychology, neuropsychiatry and pathophysiology. $\mathrm{Br} \mathcal{f}$ Psychiatry 1986;149:682-97.

44 Perry EK, Marshall E, Kerwin J, Smith CJ, Jabeen S, Cheng AV, Perry RH. Evidence of a monoaminergiccholinergic imbalance related to visual hallucinations in cholinergic imbalance related to visual hallucinations in
Lewy body dementia. $\mathcal{f}$ Neurochem 1990;55: Lewy 1454 - 6 .

45 Brown RG, Marsden CD. How common is dementia in Parkinson's disease? Lancet 1984;ii:1262-5.

46 Viitanen $M$, Mortimer JA, Webster DD. Association between presenting motor symptoms and the risk of cognitive impairment in Parkinson's disease. $\mathcal{F}$ Neurol Neurosurg Psychiatry 1994;57:1203-7. 